\title{
Partial Sheehan's Syndrome with Primary Hypothyroidism- A Delayed Diagnosis
}

\author{
NORA ANWAR KHAN, ${ }^{1}$ REZAUL EKRAM, ${ }^{2}$ PRONOB KUMAR MALLICK, ${ }^{3}$ MOHAMMAD RAFIQUL ISLAM, ${ }^{4}$ \\ MD ROBED AMIN 5
}

\begin{abstract}
:
In Sheehan;s syndrome hypopituitarism is caused by the ischemic damage to the pituitary following postpartum hemorrhage and vascular collapse. In Sheehan;s syndrome secondary hypothyroidism occurs. We report here a case of partial Sheehan's syndrome with primary hypothyroidism with high anti thyroid antibody titre which is an uncommon association.
\end{abstract}

Keywords: Sheehan's syndrome, hypopituitarism, primary hypothyroidism, secondary hypothyroidism.

\section{Introduction}

Ischemic damage to the pituitary has long been recognized as a cause of hypopituitarism. In 1914, Simmonds reported pituitary necrosis in a woman with severe puerperal sepsis and in 1937 Sheehan published his classic description of its occurrence following postpartum hemorrhage and vascular collapse. The mechanism for the ischemia in such cases is not certain. Hypotension along with vasospasm of the hypophysial arteries is currently believed to compromise arterial perfusion of the anterior pituitary. During pregnancy, the pituitary gland may be more sensitive to hypoxemia because of the hyperestrogenic state. Some degree of hypopituitarism has been reported in $32 \%$ of women with severe postpartum hemorrhage. Other investigators have noted that hypopituitarism does not always correlate with degree of hemorrhage but there is good correlation between the pituitary lesion and severe disturbances of the clotting mechanism (as in patient with placenta previa). Ischemic pituitary necrosis has been reported to occur with greater frequency in patients with diabetes mellitus. The extent of pituitary damage determines the rapidity of onset as well as magnitude of pituitary hypo function. The gland has a great secretory reserve and more than $75 \%$ must be destroyed before clinical manifestations are evident. ${ }^{1}$ Pituitary gland doesn't have ability to regenerate. Scar tissue substitutes the

1. Honorary Medical Officer, Department of Medicine, Dhaka Medical College

2. Assistant Registrar of Medicine, Department of Medicine, Dhaka Medical College

3. Registrar of Medicine, Department of Medicine, Dhaka Medical College

4. Assistant Professor of Medicine, Department of Medicine, Dhaka Medical College

5. Associate Professor of Medicine, Department of Medicine, Dhaka Medical College

Corresponding author: Dr. Md. Robed Amin, Associate Professor of Medicine, Dhaka Medical College, Bangladesh. Email: robedamin@yahoo.com, Mobile: 01711725787. necrotic cells. Presence of $50 \%$ of pituitary gland suffices for maintenance of normal functions. Partial or total hypopituitarism develops with necrosis of 70 to $90 \%$ of the gland. It is believed that $32 \%$ of women with severe post-partum bleeding develop hypopituitarism. ${ }^{2}$ The initial clinical feature in postpartum necrosis may be failure to lactate after parturition; failure to resume normal menstrual periods is another clue to the diagnosis. However, the clinical features of hypopituitarism are often subtle and years may pass before pituitary insufficiency is recognized following an ischemic insult. ${ }^{1}$ In a study of "Pituitary Autoimmunity in Patients with Sheehan's Syndrome" most of the present group of patients with Sheehan's syndrome had slow progression of their hypopituitarism with the majority presenting more than 5 years after delivery, in a state of circulatory collapse. Pituitary autoimmunity may play a role in the cause of hypopituitarism following PPH. However, slow clinical progression suggests factors other than ischemia in its pathogenesis. Tissue necrosis could release sequestered antigens, triggering autoimmunity of the pituitary and delayed hypopituitarism in Sheehan's syndrome. ${ }^{3}$

Sheehan's syndrome is usually associated with "empty sella syndrome". It occurs when the subarachnoid space extends into the sella turcica, partially filling it with CSF. The process causes remodeling and enlargement of the sella turcica and flattening of pituitary gland. The pathogenesis of primary empty sella syndrome is uncertain. It has been postulated that increased CSF pressure leads to herniation of arachnoid through the diaphragm sella. A mesenchymal defect may be present in some patients, resulting in hypoplasia of the sellar diaphragm. With this empty sella though $48 \%$ cases may present with headache but serious clinical manifestations are uncommon. ${ }^{1}$

\section{Case report}

Mrs. B. 40 years old admitted in DMCH with the complaints of unconsciousness all of a sudden followed by disorientation, vomiting, and anorexia for a couple of months. 
On examination her GCS was 8/15, BP-70/30, Pulse-100b/ min. Appearance was ill-looking, pale, cachectic. Some emergency investigations needed to run immediately which included serum electrolyte where serum sodium was low $104 \mathrm{mmol} / \mathrm{l}$, potassium was low 3. 03mmol/1, chloride was also low $83 \mathrm{mmol} / \mathrm{l}$ (Table I \& II). By giving Normal saline and injection hydrocortisone we resuscitated her properly. Within 24 hours she gained consciousness but her blood pressure was still low and postural drop was also present. On meticulous query of her obstetric history it revealed that patient's last delivery was cumbersome. Though she had a normal baby through vaginal delivery by a "daii" (means nonprofessional birth attendant) of her village at 2006. After 24 hours she had severe postpartum hemorrhage when she was taken to a local hospital where they found retained placenta and did a D\&C (dilatation and curettage). Later on she was having a difficulty in breast feeding her child due to lactational insufficiency but not failure. She has three children; all of them were born via normal vaginal delivery before this one. Further obstetric history also elaborates that she suffered from oligomenorrhoea for 6 years from 2006 onwards and from 2013 she has total amenorrhoea. All through these years she was having loss of libido.

On examination she was pale looking, lethargic, fine wrinkled skin, postural drop of blood pressure(on lying 90/50 and on standing 70/40) was found, skin changes of hand, body, legs were dry, coarse and wrinkled, breast started to atrophy, axillary hairs were lost. On investigation of complete blood count revealed normal with white blood cell count-11 thousands/cumm, rbc-3. 75 million/cumm, platelet count-2 lac 55 thousands/cumm, hemoglobin-11 g/dl, ESR-60 mm in $1^{\text {st }}$ hour.

Majority of her anterior hormone panel (ACTH, Growth hormone) were low except serum TSH which was mildly raised and serum FSH, LH which was lower level of normal limit. But free T3 and free T4 were low. Serum cortisol level was low evidenced by short synacten test. Serum aldosterone was in lower level of normal limit. MRI of her brain (Figure 1) showed 'partial empty sella'. She was given supplementation of hydrocortisone $10 \mathrm{mg}$ tablet one in the morning and half dose at $3 \mathrm{pm}$ with tablet thyroxin replacement daily. On discharge she was given steroid card and she was leveled as "Partial Sheehan's Syndrome with primary hypothyroidism".
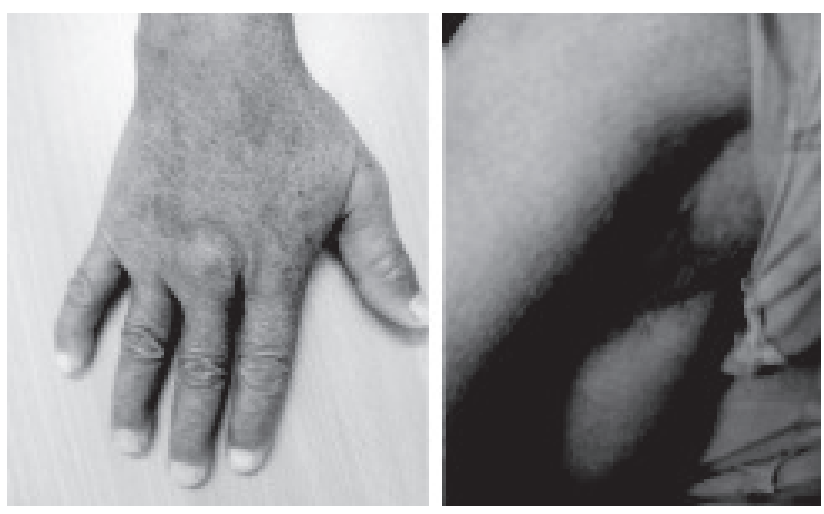

Fig : 1\&2: Hands showing dry, coarse skin and also fine wrinkling are visible; Axillary hairs are completely lost (copy right-Nora anwar khan)

Table I

Electrolyte profile.

\begin{tabular}{lcccc}
\hline Electrolyte & $26-2-15$ & $27-2-15$ & $30-2-15$ & $2-3-15$ \\
\hline $\mathrm{Na}$ & 104 & 112 & 120 & 135 \\
$\mathrm{~K}$ & 3.38 & 3.45 & 3.5 & 3.54 \\
$\mathrm{Cl}$ & 68 & 78 & 83 & 98 \\
$\mathrm{HCO} 3$ & 30 & 28 & 28 & 28 \\
\hline
\end{tabular}

Table II

Biochemical profile.

\begin{tabular}{lll}
\hline Serum cortisol(basal) & $0.75 \mathrm{ug} / \mathrm{dl}$ & Comment-low \\
Serum cortisol (after short synacthen test) & $1.14 \mathrm{ug} / \mathrm{dl}$ & Comment-low \\
ACTH & $11.4 \mathrm{pg} / \mathrm{ml}$ & Comment-low \\
FSH & $13.27 \mathrm{miu} / \mathrm{ml}$ & Comment-lower limit of normal level \\
LH & $4.67 \mathrm{miu} / \mathrm{ml}$ & Comment-lower limit of normal level \\
Growth Hormone & $0.072 \mathrm{ng} / \mathrm{ml}$ & Comment-low \\
TSH & $6.70 \mathrm{miu} / \mathrm{ml}$ & Comment- raised \\
Free T3, Free T4 & $1.10 \mathrm{pg} / \mathrm{ml} .74 \mathrm{ng} / \mathrm{dl}$ & Comment-both are low \\
Serum cortisol(basal) & $0.75 \mathrm{ug} / \mathrm{dl}$ & Comment-low \\
Anti Thyroperoxidase (Anti TPO) Antibody & $1400 \mathrm{IU} / \mathrm{ml}$ & Comment-very high \\
Anti-thyroglobulin Antibody & $960 \mathrm{IU} / \mathrm{ml}$ & Comment-very high \\
\hline
\end{tabular}




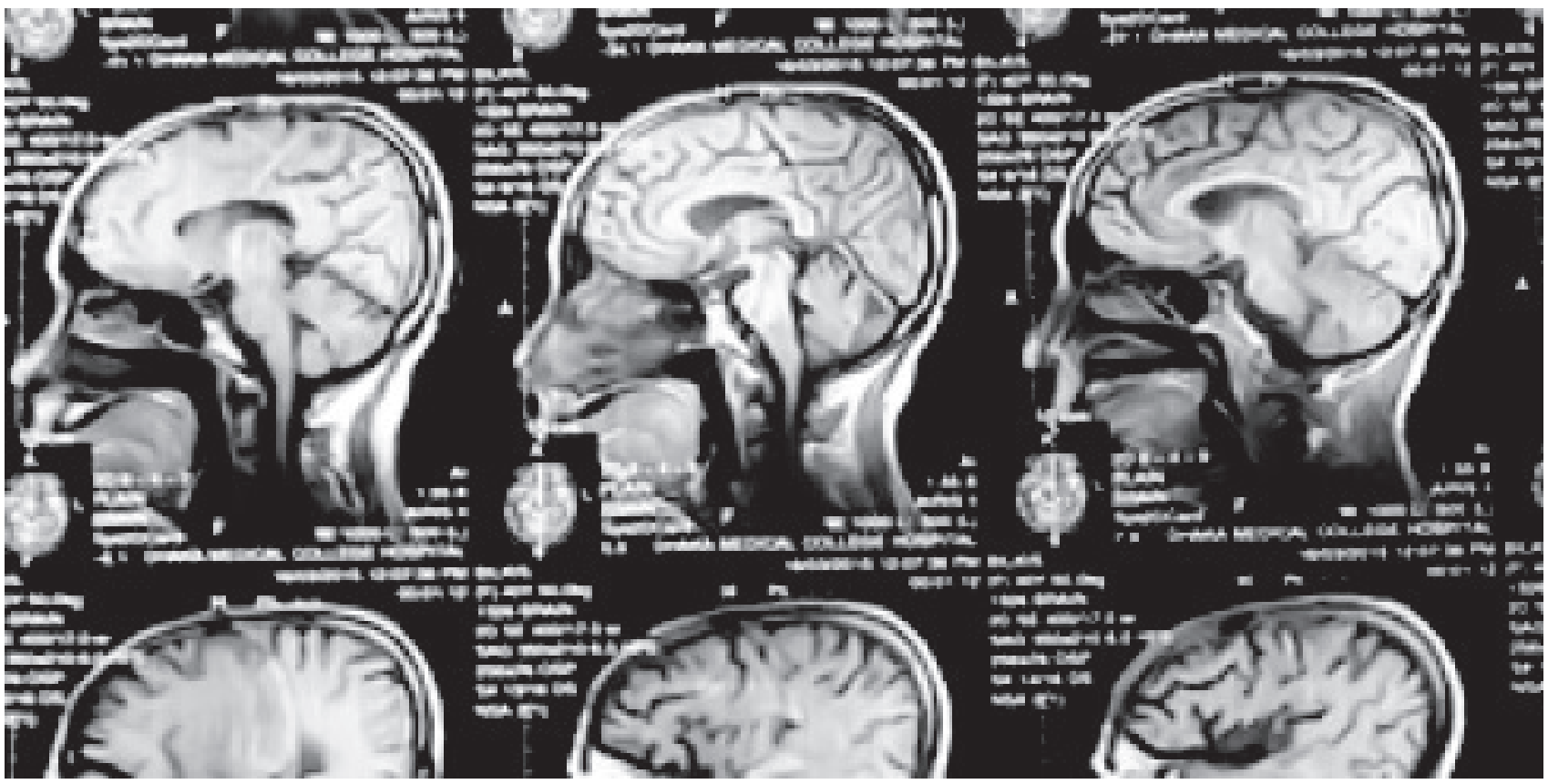

Fig 1: MRI of brain showing empty sella

\section{Discussion}

The pathogenesis of pituitary failure following PPH is not clear. Sheehan and Davies proposed vasospasm of the pituitary vessels and consequent ischemic necrosis of the pituitary. However, experimental evidence suggests efficient adaptive autoregulation of adeno-hypophyseal blood flow during hypotension caused by acute blood loss. Massive or submassive ischemic necrosis of the pituitary should result in acute pituitary failure in Sheehan's syndrome to that observed in pituitary apoplexy. Maccagnan et al. assessed pituitary function among patients with pituitary apoplexy managed conservatively. Two weeks following apoplexy, gonadotropin, thyrotropin, and corticotropin deficiency were observed in $80 \%, 50 \%$, and $33 \%$, respectively. However, in the present study, patients survived acute blood loss and related pituitary failure for several years without any hormone replacement. Despite lactational failure and amenorrhea, indicating pituitary insufficiency dating from the postpartum period, they did not seek medical attention until lifethreatening symptoms of circulatory collapse because of adrenal insufficiency supervened. This sequence of loss of pituitary trophic hormone function suggests that destruction of the pituitary gland was partial from the inception with progressive loss over time involving thyrotroph and corticotroph function. Such delayed presentation in patients with Sheehan's syndrome could be due to the subtlety of symptomatology and thus the associated failure to recognize hypopituitarism. There are two isolated case reports of pituitary autoimmunity in Sheehan's syndrome. In 1965
Engelberth and Jezkova, using a complement consumption test reported PitAb in a patient with Sheehan's syndrome. In 1982 Pouplard observed PitAb by indirect immunofluorescence in three of four patients with Sheehan's syndrome. However, in 1969 Nerup et al. could not demonstrate PitAb in any of six patients with Sheehan's syndrome. The variable reports on PitAb positivity in Sheehan's syndrome could be due to the small numbers of patients studied or technical issues related to the immunofluorescence assays used. In the study done in university of Newcastle of New South Wales on pituitary autoimmunity of patients with sheehans syndrome, 63. 1\% of patients with Sheehan's syndrome had PitAb against a 49-kDa pituitary cytosolic antigen, even 5 years after the onset of illness. However, only $14.2 \%$ of patients in the other group had PitAb positivity. This trend of higher prevalence of pituitary autoantibody in the Sheehan's syndrome patients with PPH suggests a relationship between the PPH and PitAb positivity. However, the difference observed in PitAb positivity between the two groups did not attain statistical significance. The comparable prevalence of TMA positivity among the study groups and controls showed no increase in the prevalence of thyroid autoimmunity in the group of Sheehan's syndrome(SS) patients with PitAb positivity. ${ }^{3}$ Postpartum pituitary necrosis is a known complication, but it is now rarely seen in developed countries. Even if postpartum hemorrhage has been well managed, this complication cannot be excluded, and it can be life threatening. It is necessary to consider this diagnosis in all 
patients having presented with a cardiovascular collapse during childbirth, whatever the cause, and in the presence of the classic signs of pituitary insufficiency. In some cases the pituitary necrosis is only partial and the syndrome can present in atypical and incomplete forms further complicating the diagnostic procedure. Hypopituitarism has several possible etiologies. Apart from adenohypophysal necrosis, other causes are quoted: tumoral, immunological, iatrogenic, traumatic, infectious and genetic. ${ }^{4} \mathrm{SS}$ is a very significant cause of maternal morbidity and mortality in developing countries although it is a rarity in developed countries in which obstetrical care has been improved. In a study of Firat University School of Medicine, Firat Medical Center, Department of Endocrinology and Metabolism, 23119Elaz1, Turkey, reviewed 20 cases retrospectively who were diagnosed as SS in their clinic. The patients aged 40 to 65 years with a mean age of $51.12 \pm 9.44$ years (mean \pm SD). Time to make a definitive diagnosis of the disease ranged between 5 and 25 years with a mean of $16.35 \pm 4.74$ years. Three of the patients $(15 \%)$ had a previous diagnosis of SS. Three patients $(15 \%)$ were referred to emergency service for hypoglycemia, three patients $(15 \%)$ for hypothyroidism and one patient $(5 \%)$ for hyponatremia. Dynamic examination of the pituitary revealed GH, Prolactin, FSH, TSH and ACTH insufficiency in all of the patients. One patient had a sufficient LH response to LHRH challenge. All of the patients were imaged with pituitary MRI. Eleven patients had empty sella and 9 patients had partial empty sella. $^{2}$

Our patient had PPH on 2006 and she presented in our hospital with severe hyponatremia with circulatory collapse but not hypoglycemia. So it took almost 9 years to have florid and acute life-threatening manifestations of hypopituitarism.
She was also diagnosed in our hospital as hypothyroid. During all these 9 years gradually she developed lactation insufficiency, weakness, fatigue, lethargy, anorexia, loss of libido, oligomenorrohea to amenorrhoea. All anterior pituitary hormone panel was either low or lower limit of normal value except TSH which is raised. Later we also have done the autoantibody for hypothyroidism (Anti TPO antibody and Anti thyoroglobulin antibody) to see the autoimmune status. Both Anti TPO antibody and Anti thyoroglobulin antibody were very high which indicates "Partial Sheehan's Syndrome with Primary Hypothyroidism". Pituitary autoantibody is not possible in our settings due to lack of facility. Sheehan's Syndrome is still a problem in our country which is diagnosed late, especially in rural areas. Considering the duration of disease, important delays occur in diagnosis and treatment of the disease.

\section{Conflicts of Interest: None.}

\section{References}

1. Greenspan F, Baxter J. D, Endocrinology evaluation of the hypothamamic pituitary axis-basic and clinical endocrinology $4^{\text {th }}$ edition: 91-93.

2. Özkan Y \& Colak R. Sheehan Syndrome: Clinical and laboratory evaluation of 20 cases-Neuroendocrinology Letter 2005;26(3):257-260.

3. Goswami R, Kochupillai N, Patricia A. Crock, Jaleel A, Gupta N; Pituitary Autoimmunity in Patients with Sheehan's Syndrome-The Journal of Clinical Endocrinology \& Metabolism 87(9):4137-4141.

4. Errarhay S, Kamaoui I, Bouchikhi C, Châara H, Bouguern H, Tizniti S, Melhouf A, and Banani A; Sheehan's Syndrome A Case Report and Literature Review-LJM; www. ljm.org.ly 